Special issue of the 3rd International Conference on Computational and Experimental Science and Engineering (ICCESEN 2016)

\title{
Analysis of Out of Control Signals in Multivariate Processes with Multilayer Neural Network
}

\author{
S. BORAN* AND D.D. DIREN \\ Sakarya University, Department of Industrial Engineering, Sakarya, Turkey
}

\begin{abstract}
Control charts that are used for monitoring the process and detecting the out-of-control signals are important tools for statistical process control. It is simple to estimate source(s) for out-of-control signals in the univariate process, whereas it is difficult to identify the source(s) in the multivariate processes. The reason is that these kinds of processes require monitoring and controlling of more than one quality characteristics simultaneously. In this study, the proposed model is expected to detect the source(s) for out-of-control signals without help of an expert in the process, by using a multilayer neural network. This model was implemented in furniture fasteners manufacturing. Time gain was obtained while detecting source(s) for out-of-control signals.
\end{abstract}

DOI: 10.12693/APhysPolA.132.1054

PACS/topics: 07.05.Mh, 02.70.Rr

\section{Introduction}

It has become difficult for companies to survive in today's competitive environment. Quality is one of the most important components for success in production. Some techniques have been developed for providing the desired quality. One of the most important techniques is quality control charts. These charts provide monitoring process and detect the source(s) of out-of-control signals. In multivariate processes, control charts are used for determining the out-of-control signals. The main problem with this method is when the number of variables is more than one; every variable has its own control charts that should be used simultaneously. This leads to great loss of time and labor.

The sources may also depend on a variable(s) and/or the relationship between variables. Control charts that detect the out-of-control signals are generally created with $\mathrm{T}^{2}$ statistics. However, with this chart, it is accepted that there is interaction between variables.

In this study, a multilayer neural network model has been established for eliminating disadvantages caused by separate evaluation of the variables. Our proposed model is based on individual (I) control charts, because there was no relationship between variables.

\section{Methods}

\subsection{Univariate and multivariate control chart}

Control chart, developed by Shewhart in 1924, is an important monitoring tool for detecting the deviation of one variable in the process. The univariate control diagrams are varying according to the characteristics of the variables in the process or the sample size. Typically, there is a centerline, normal values of process, upper control limit (UCL) and lower control limit (LCL) on the

\footnotetext{
*corresponding author; e-mail: boran@sakarya.edu.tr
}

chart [1]. If the sample size is one, individual (I) and moving range (MR) control charts are used. The upper and lower control limit equations for the (I) control chart are as follows [2]:

$$
\begin{aligned}
& \mathrm{UCL}=\bar{X}+3 \frac{\overline{\mathrm{MR}}}{d_{2}}, \\
& \text { Center line }=\bar{X}, \\
& \mathrm{LCL}=\bar{X}-3 \frac{\overline{\mathrm{MR}}}{d_{2}}, \\
& \mathrm{MR}_{i}=\left|x_{i}-x_{i-1}\right| .
\end{aligned}
$$

where $\bar{X}$ is mean, $d_{2}$ is 1.128 and MR bar is mean of moving range.

If multiple variables, affecting the process, are monitored simultaneously, then multivariate quality control diagrams are used [2]. Hotelling $\mathrm{T}^{2}$ control diagram, developed by Hotelling in 1947, is the oldest multivariable control diagram and is based on arithmetic average [3]. Although it is possible to evaluate the variables together and detect out-of-control signals in the process, it is not possible to determine the source(s) for these signals using this chart.

Methods, which have been developed for identifying the sources, can be grouped into two main categories: statistical methods and artificial neural networks. Statistical methods are based on decomposition. $\mathrm{T}^{2}$ decomposition method, developed by Mason et al. (1995), contains all variables and groups them into conditional and unconditional components [4]. Thus, it determines the variable(s). There are many studies about detecting the sources for out-of-control signals by using $\mathrm{T}^{2}$ decomposition method and some studies are related to definition of conditional and unconditional components for different number of variables with this method [4-9].

\subsection{Artificial neural network}

Neural networks, unlike traditional computer algorithms, contain different learning algorithms and have the 
shape of nerve cells in human brain, which are mathematically modelled with computer systems [10]. Artificial neural networks (ANNs), the most basic components of neural networks, have processing systems that can easily learn the behavior of complex systems. ANNs have the ability to establish relationships between the inputs and outputs and produce results. ANN can be trained with current examples to model the problem and the results can be used in real time [11-14].

Multilayer neural network is the most common method, used for forecasting problems (Fig. 1). This type of neural networks has an input layer, an output layer and one or more hidden layers, which is called feedforward [15].

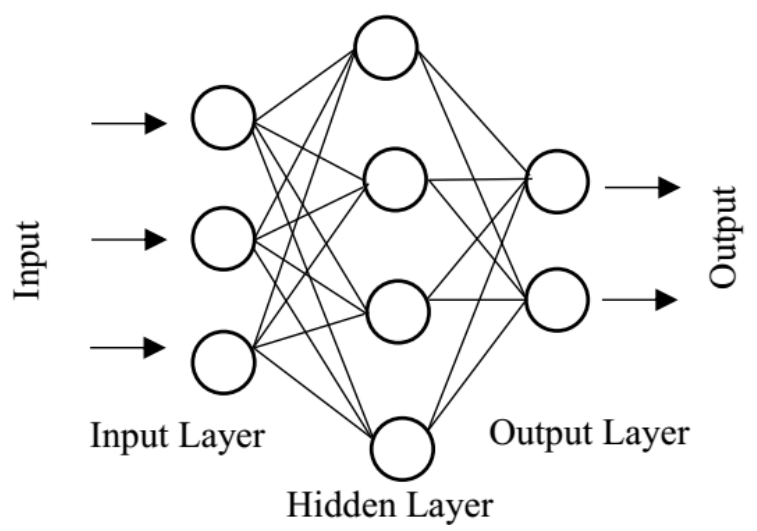

Fig. 1. The structure of the multilayer neural network.

ANN is frequently used in analysis of out-of-control signals. Studies on identifying the variable(s) with ANN can be grouped into two main categories, according to pattern number [5, 16-20] and pattern type [21-26].

\section{Proposed model}

The steps of proposed model are as follows:

- Determining and measuring the variables,

- Forming of an individual (I) control chart for every variable,

- Determining out-of-control condition for every control chart,

- Designing multilayer neural network model, based on values of (I) control charts.

- Obtaining the source(s) for out-of-control signals with this designed neural network

This network has an input of the number of variables $p$ that affect the process. There is an output for every variable. Output values are 0 or 1 . "Zero" means incontrol condition while "one" means out-of-control condition. Output includes number of $2^{p}-1$ classes and the network has hidden layers. Number of neurons in the hidden layer is found by trial.

\section{Results and discussion}

This proposed model was implemented to furniture fasteners, manufactured in ABC Company, operating in Turkey (Fig. 2) the production process of the product is as follows: drilling, cutting and bending.

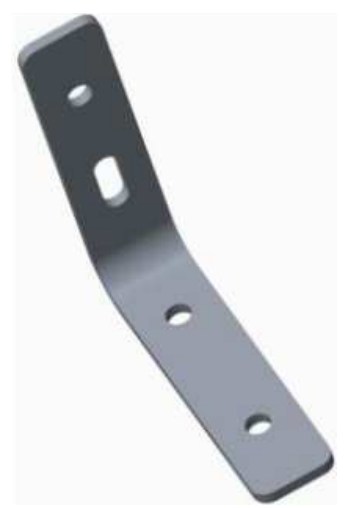

Fig. 2. Furniture fastener product.

Four quality variables have been chosen, as follows; $x_{1}$ : first diameter, $x_{2}$ : second diameter, $x_{3}$ : the length of the part, and $x_{4}$ : bending grade. Firstly, 500 data records were randomly generated by simulation, according to mean, variance and the measurements on technical drawing.

Because the variables are independent and cannot be monitored simultaneously, individual (I) control chart is used as shift detector. Limits are determined according to this detector. Individual (I) control chart was applied to variables one by one. Control limits and means of variables are shown in Table I. The variables were examined separately with (I) control chart. It was found that 136th and 139th samples on the first variable, 143rd sample on the third variable, 149th sample on the fourth variable were out-of-control. Then, multivariate neural network model was developed for evaluating the variables together and for detecting the variable(s) (source(s)) of out-of-control signals. This network has an input layer consisting of four neurons, hidden layer consisting of sixteen neurons and an output layer consisting of four neurons. Each neuron in output layer represents an output class. Outputs are as follows: $(0,0,0,0),(1,0,0,0)$, $(0,1,0,0),(0,0,10),(0,0,0,1),(1,1,0,0),(1,0,1,0),(1,0,0,1)$, $(0,1,1,0), \ldots(1,1,1,1)$. For example, $(1,0,0,1)$ means; $x_{1}$ : out-of-control, $x_{2}$ : in-control, $x_{3}$ :in-control, and $x_{4}$ : out-of-control.

The network generates the outputs by using individual control charts limits. The structure of neural network is shown in Fig. 3.

The structure of the model is a back-propagation, feedforward multilayer neural network. It was chosen due to its ease of use and high prediction success.

The network was trained with sufficient data by using MATLAB computer software. The input data were normalized before being processed and reduced to the $[0,1]$ 


\section{TABLE I}

The lower and upper control limits for the I control charts.

\begin{tabular}{c|c|c|c}
\hline \hline Variables & Mean & UCL & LCL \\
\hline$x_{1}$ & 8 & 7.510 & 8.491 \\
$x_{2}$ & 7 & 6.479 & 7.535 \\
$x_{3}$ & 150 & 148.960 & 151.056 \\
$x_{4}$ & 90 & 87.978 & 92.051
\end{tabular}

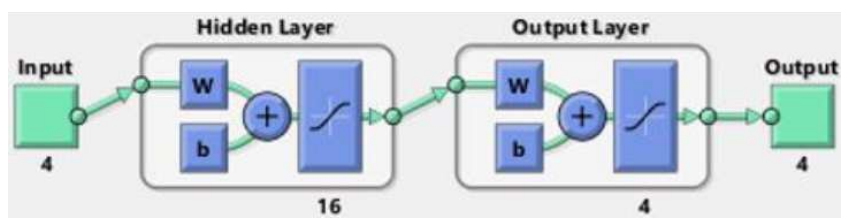

Fig. 3. The structure of neural network.

range. $70 \%$ of the data were reserved for training, $15 \%$ for validation and $15 \%$ for the test. Weights were randomly assigned for training.

Number of neurons in the hidden layer was found by trying. The performance function of the model is mean squared error (MSE). The training performance of neural network is shown in Fig. 4.

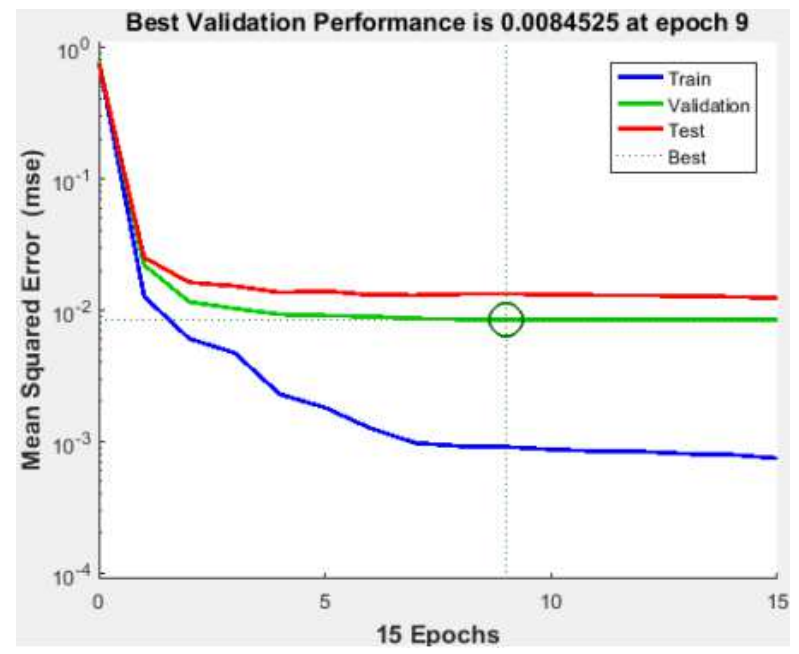

Fig. 4. Training performance of neural network.

The training process stopped when the MSE got to value of $10^{-3}$. MSE value showed the adequacy of model. After training, the trained network was simulated for a dataset containing 150 samples and output values were obtained. It must be specified that in cases, when the output values are very close 0 or 1 , they were rounded to 0 or 1 .

When result of model was examined, it was seen that 49 th, 136th, 139th, 143rd, and 149th samples were outof-control. The results of the variables of out-of-control conditions, obtained from the neural network model, are shown in Table II.
TABLE II

Results of the out-of-control conditions produced by multilayer neural network.

\begin{tabular}{c|c}
\hline $\begin{array}{c}\text { Out-of-control } \\
\text { sample number produced by } \\
\text { multilayer neural network }\end{array}$ & $\begin{array}{c}\text { Out-of-control } \\
\text { variable }\end{array}$ \\
\hline 49 & $x_{2}$ and $x_{3}$ \\
136 & $x_{1}$ \\
139 & $x_{1}$ \\
143 & $x_{3}$ \\
149 & $x_{4}$
\end{tabular}

It can be seen that the 49th sample was found to be out-of-control by using neural network. The variables of the out-of-control conditions are the 2 nd and the $3 \mathrm{rd}$ variables. However, it can be seen that 49th sample was in-control on (I) control chart. The variables are independent, but there can be an interaction between two variables.

\section{Conclusions}

This study presents a multilayer perceptron network model for out-of-control condition analysis in multivariate variable processes. In individual (I) control chart method, every variable has its own control chart and variables are considered as independent. With the proposed model, a large number of variables, affecting real processes can be analyzed together. Hence, the loss of time and labor are eliminated. For these reasons, multilayer neural network is considered to be an effective tool.

\section{References}

[1] M.-H. Shu, H.-C. Wu, Comput. Ind. Eng. 61, 676 (2011).

[2] D.C. Montgomery, Introduction to Statistical Quality Control, 6th ed., John Wiley \& Sons, New York 2001.

[3] H. Hotelling, in: Techniques of Statistical Analysis, Eds. C. Eisenhart, M.W. Hastay, W.A. Wallis, McGraw Hill, New York 1947, p. 111.

[4] R.L. Mason, N.D. Tracy, Y.C. Young, J. Qual. Technol. 27, 99 (1995).

[5] F. Aparisi, A. Gerardo, J. Sanz, IIE Trans. 38, 647 (2006).

[6] J. Li, J. Jin, J. Shi, J. Qual. Technol. 40, 46 (2008).

[7] M.R. Pina-Monarrez, Int. J. Eng.-Theory 20, 401 (2013).

[8] N.S. Agog, H.C. Dikko, O.E. Asiribo, Int. J. Innov. Res. Sci Eng. Tech. 3, 449 (2014).

[9] A.A. Akeem, A. Yahaya, O. Asiribo, Am. J. Theor. Appl. Stat. 4, 432 (2015).

[10] B. Warner, M. Misra, Am. Stat. 50, 284 (1996).

[11] R. Tuntas, Acta Phys. Pol. A 128, B-78 (2015).

[12] E. Boutalbi, L. Ait Gougam, F. Mekideche-Chafa, Acta Phys. Pol. A 128, B-271 (2015). 
[13] M. Davraz, Ş. Kilinçarslan, H. Ceylan, Acta Phys. Pol. A 128, B-184 (2015).

[14] Y. Özcanli, F. Kosovali Çavuş, M. Beken, Acta Phys. Pol. A 130, 444 (2016).

[15] M. Negnevitsky, Artificial Intelligence: A Guide to Intelligent Systems, 2nd ed., Addison Wesley, 2005.

[16] S. Taghi, A. Niaki, B. Abbasi, Qual. Reliab. Eng. Int. 21, 825 (2005).

[17] C.-S. Cheng, H.-P. Cheng, Expert. Syst. Appl. 35, 198 (2008).

[18] T.T. El-Midany, M.A. El-Baz, M.S. Abd-Elwahed, Expert. Syst. Appl. 37, 1035 (2008).

[19] T.-F. Li, S. Hu, Z.-Y. Wein, Z.-Q. Liao, Math. Probl. Eng. 2, 9 (2013).
[20] S. Du, J. Lv, L. Xi, Int. J. Prod. Res. 50, 6288 (2012).

[21] C.-H. Wang, T.-P. Dong, A. Kuo, J. Intell. Manuf. 20, 409 (2009).

[22] X. Wang, in: Int. Conf. Computational Intelligence and Security, (2008), p. 238.

[23] J. Yu, L. Xi, X. Zhou, Eng. Appl. Artif. Intel. 22, 141 (2009).

[24] C.-J. Lu, Y.E. Shao, P.H. Li, Neurocomputing 74, 1908 (2011).

[25] W.-A. Yang, J. Intell. Manuf. 26, 769 (2015).

[26] I. Masood, A. Hassan, Int. J. Adv. Manuf. Technol. 9, 1201 (2013). 